http://jmscr.igmpublication.org/home/

ISSN (e)-2347-176x ISSN (p) 2455-0450

crossref DOI: https://dx.doi.org/10.18535/jmscr/v9i7.18

Journal Of Medical Science And Clinical Research

IGM Publication

An official Publication of IGM Publication

\title{
Observational Study of Lipid Profile in Hypothyroid Patients in Secondary Health Institution in Himalayan Region
}

\author{
Authors \\ Dr Parul Bhardwaj ${ }^{1}$, Dr Shivbrat Sharma ${ }^{2}$ \\ ${ }^{1}$ MD Internal Medicine, Civil Hospital Dehra (H.P.) \\ ${ }^{2}$ Junior Resident ENT, DRPGMC Kangra at Tanda (H.P.) \\ *Corresponding Author \\ Dr Shivbrat Sharma \\ Junior Resident, MS ENT, DRPGMC Kangra at Tanda (H.P.), India
}

\begin{abstract}
Thyroid dysfunction has a great impact on lipids as well as a number of other cardiovascular risk factors. Hypothyroidism is relatively common and is associated with an unfavorable effect on lipids. Substitution therapy is beneficial for patients with overt hypothyroidism, improving lipid profile. The present study aims to assess the association of hypothyroidism with lipid abnormalities.
\end{abstract}

\section{Introduction}

Hypothyroidism is a common metabolic disorder in the general population. Indeed, data from the third National Health and Nutrition Examination Survey (NHANES III) showed a $4.6 \%$ prevalence of hypothyroidism in the general population, while $9.5 \%$ of the Colorado prevalence study participants had elevated levels of $\mathrm{TSH}^{1}$. Thyroid failure is more common in women and its prevalence rises with age. Hypothyroid patients have increased levels of TC and LDL-C ${ }^{1}$. Indeed, hypothyroidism is a common cause of secondary dyslipidemia $^{2}$.

Hypothyroidism is defined as a deficiency of thyroid activity, which results from reduced secretion of both $\mathrm{T} 3$ and $\mathrm{T} 4$ irrespective of the cause $^{3}$.

Clinically hypothyroidism may present with variety of symptoms and signs involving major systems of the body like endocrine, cardio vascular, central nervous system, musculo skeletal, heamatological, reproductive, gastrointestinal and dermatological ${ }^{4}$.

Thyroid hormones have significant effects on synthesis, mobilization and metabolism of lipids. Overt hypothyroidism is associated with significant increase in circulating concentrations of total LDL-Cholesterol leading to coronary artery disease. Hypercholestrolemia is favoured due to the hormone deficit and to the decreased activity of lipoprotein lipase $\mathrm{e}^{5,6}$.

The present study aims to assess the association of hypothyroidism with lipid abnormalities.

It has been postulated that hypothyroidism would increase the risk for cardiovascular disease, due to reported association between hypothyroidism and hyperlipidimia as well as potential associations with other cardiovascular risk factors such as abnormal endothelial reactivity, enhanced LDL 
oxidation,

inflammation

and

hyperhomocysteinemia ${ }^{7}$.

\section{Materials and Methods}

Total 66 patients with biochemically confirmed hypothyroidism attending medicine OPD in Civil hospital Dehra from August 2020 to April 2021 meeting inclusion and exclusion criteria were included in the study after obtaining informed consent.

\section{Inclusion Criteria}

1) All patients more than 18 years of age with diabetes mellitus.

2) All patients with hypothyroidism willing to participate in this study.

\section{Exclusion Criteria}

Patients who had secondary causes of hypothyroidism like

- Radioiodine ablation

- On antithyroid drugs

- Had undergone thyroid surgery.

Patients with end stage renal disease, post myocardial infarction, congestive cardiac failure, type II diabetes mellitus, patients on anti lipidemic drugs and thyroid hormone therapy, women on oral contraceptive pills were excluded from the study.

\section{Methology}

Blood samples of patients were drawn after an overnight fasting in serum tube. Serum was separated for the estimation for the estimation of serum TSH, TT3, TT4 and total cholesterol, triglyceride, high density lipoprotein, low density lipoprotein which were derived from TC and TG using FRiewald's formula [LDL $=\mathrm{TC}-(\mathrm{HDL}+$ TG/5)]. TC was estimated with CHOD PAP method, TG and HDL were estimated using the enzymatic colorimetric method. T3, T4, TSH and anti TPO antibody were estimated by using DIASORIN immunoassay LIAISON analyser. This machine does the quantitative determination of TSH in human serum via chemiluminescence immune assay method.
Normal references values are as follows: Total cholesterol: 100-200mg/DL,TG: 150-199mg/dl, LDL:100-130mg/dl, HDL:40-60mg/dl,TT3; 60200ng/dl,TT4: 5-12ug/dl,TSH:0.3-4.12ug/dl.

\section{Results}

Total 66 patients with hypothyroidism were enrolled in the study with 49 patients $(74.2 \%)$ were subclinical hypothyroidism and 17 (25.8\%) were overt hypothyroidism. Out of 66, $28(42.42 \%)$ were male and $38(57.58 \%)$ were females. The mean age of the patients was $58.15( \pm$ $9.53)$ with minimum were 32 and maximum were 78. Maximum patients 27 were in 51-60 years age group followed by 20 in 61-70 year age group. The mea total cholesterol was $196.56 \pm 87.08$ with minimum were 60 and maximum were 733.34 patients $(51.5 \%)$ had total cholesterol was $>200 \mathrm{mg} / \mathrm{dl}$ and 32 patients had total cholesterol was $48.5 \%$. The mean TG was $177.67 \pm 114$ with minimum were 57 and maximum were 908. 28 patients $(42.4 \%)$ had $\mathrm{TG}<150 \mathrm{mg} / \mathrm{dl}$ and 38 patients (57.6\%) had TG $>150 \mathrm{mg} / \mathrm{dl}$. Mean HDL was $44.11 \pm 11.99$ with minimum was 13 and maximum was 80.30 patients $(45.5 \%)$ had $\mathrm{HDL}<40 \mathrm{mg} / \mathrm{dl}$ and 36 patients $(54.5 \%)$ had HDL > 40mg/dl. Mean LDL was 138.63 \pm 31.51 with minimum 77 and maximum 265. 40 patients $(60.60 \%)$ had LDL $>130 \mathrm{mg} / \mathrm{dl}$ and $26(39.4 \%)$ patients had LDL $<130 \mathrm{mg} / \mathrm{dl}$.

\section{Discussion}

Thyroid disorders are known to influence lipid metabolism. In our study, total 66 patients with hypothyroidism were included. In our study hypothyroidism was more common in females and in elderly population which is similar to the study conducted by Kebria et $\mathrm{al}^{8}$. In our study the patients with hypercholesterolemia with hypothyroidism were more than normal cholesterol levels similar to study done by this can be explained due to fact that hypercholesterolaemia in hypothyroidism occur due to reduced catabolism of lipoproteins, that 
results from the decrease expression of lipoprotein receptors and LDL cholesterol ${ }^{9}$. The TG levels were high in hypothyroidism patients similar to this study ${ }^{8}$. This can also be explained due to decrease levels of lipoprotein lipase which results in decrease clearance of triglyceride rich lipoproteins ${ }^{9}$. Maximum patients with hypothyroidism high HDL levels which can be due to decreased activity of cholesterol ester transfer protein which results in reduced transfer of cholesterol esters from HDL to VLDL ${ }^{10}$. The LDL levels were found also high in hypothyroidism patients similar to the study which can be occur due to increase s in large LDL particle $^{11}$. Hypothyroidism with high LDL levels increases the risk for atherosclerosis.

\section{Conclusion}

In our study patients with hypothyroidism have dyslipidimia which further can increases the cardiovascular risk. Hence patient with hypothyroidism must be evaluated for lipid abnormality to prevent further cardiovascular risks.

\section{References}

1. Canaris GJ, Manowitz NR, Mayor G, Ridgway EC. The Colorado thyroid disease prevalence study. Arch Intern Med. 2000;160:526-34.

2. Stone NJ. Secondary causes of hyperlipidemia. Med Clin North Am. 1994;78:117-41.

3. Shaikh BA. Clinical features of primary hypothyroidism: a year experience at Chandka medical college, Larkana. Medical Channel. 2008:14 (1) : 72-75.

4. Toft A. Hypothyroidism. Med Intern 1989; 8:2596-2600.

5. Cappola AR, Ladenson PW. Hypothyroidism and atherosclerosis. Journal of Clinical Endocrinology. Metabolism.2003; 88:2438-2444

6. Jawed S, Khawaja TF, Sultan A. Mahmood. Alterations in lipid profile in old age hypothyroid patients. Ann. King Edward Med. Coll. Sep. 2005; 11(3):311313.

7. Nyirenda MJ, Clark DN, Finlayson AR, Read J, Elders A et al. 2005. Thyroid disease and increased cardiovascular risk. Thyroid 15:718-724.

8. Kibria KG, Alam MR, Paul HR, Fariduddin $M$ et al. Pattern of Dyslipidemia in Hypothyroid Patients -A cross sectional study. BSMMU J.2013;6(2):156-160.

9. Stone NJ. Secondary causes of hyperlipidemia. Med Clin North Am. 1994;78:117-114.

10. Dullart RP, Hoogenberg K, Groener JE et al. The activity of cholesterol ester transfer protein is decreased in hypothyroidism: a possible contribution to alterations in high -density lipoproteins. Eur J Clin Invest. 1990;20:581-7.

11. Abbas JM, Chakraborty J, Akanji AO, Doi SA. Hypoyhroidism results in small dense LDL independent of IRS traits and hypertriglyceridemia. Endocr J.2008; 55:381-9. 